\title{
Synthesis and characterization of zinc ferrite nanoparticles obtained by self-propagating low-temperature combustion method
}

\author{
P M PRITHVIRAJ SWAMY, S BASAVARAJA ${ }^{1}$, ARUNKUMAR LAGASHETTY ${ }^{2}$, \\ N V SRINIVAS RAO ${ }^{3}$, R NIJAGUNAPPA ${ }^{4}$ and A VENKATARAMAN* \\ Department of Materials Science, Gulbarga University, Gulbarga 585 106, India \\ ${ }^{1}$ Present address: Veeco-India Nanotechnology Laboratory, \\ Jawaharlal Nehru Centre for Advanced Scientific Research, Bangalore 560 064, India \\ ${ }^{2}$ Appa Institute of Engineering and Technology, Gulbarga 585 102, India \\ ${ }^{3}$ R\&D Centre, Premier Explosives Pvt. Ltd., Hyderabad 500 015, India \\ ${ }^{4}$ Department of Environmental Sciences, Gulbarga University, Gulbarga 585 106, India
}

MS received 14 April 2010

\begin{abstract}
The self-propagating low-temperature combustion method was used to produce nanocrystalline particles of zinc ferrite. The products were characterized for chemical and phase composition, morphology and magnetic properties. The results obtained showed the formation of single-phase zinc ferrite nanoparticles with an average particle size of about $40 \mathrm{~nm}$. As-synthesized powder displayed good magnetic property. Due to the simplicity and low cost of this process, it could also become a valuable starting point for the generation of other mixed and complex ferrites.
\end{abstract}

Keywords. Zinc ferrite; self-propagating combustion method; magnetic property; X-ray diffraction; morphology.

\section{Introduction}

In recent years, nanosized materials have been extensively studied by researchers worldwide because of their unique physical properties, such as electrical conductivity, optical bandgap, refractive index and magnetic properties, and superior mechanical properties such as hardness of nanomaterials and chemical properties compared with their counterpart bulk materials (Martin 1994). As the research community is gaining a deeper understanding of these unique behaviours and applications of nanostructured materials, a new field of study known as 'nanotechnology' has emerged (Bhattacharyya et al 2001; Kondic and Diez 2009). Among various nanomaterials, mainly spinel ferrite $\left(\mathrm{MFe}_{2} \mathrm{O}_{4}, \mathrm{M}=\mathrm{Ni}, \mathrm{Co}, \mathrm{Mn}, \mathrm{Zn}\right.$, etc.) nanoparticles have become immensely popular magnetic materials for a wide variety of applications such as electronic ignition systems, generators, vending machines, medical implants, wrist watches, inductor core, transformer circuits, magnetic sensors and recording equipment, telecommunications, magnetic fluids, microwave absorbers and other high-frequency applications (Snelling 1989; Jiles 1991; Willard et al 2004; Mathew and Jiang 2007). A distinct merit of ferrites over other magnetic materials such as

*Author for correspondence (raman_chem@ @rediffmail.com) iron and metallic alloys is their high electrical resistivity due to which ferrites perform much better at high frequencies. High electrical resistivity prevents induction of eddy currents and the resultant loss of energy. High permeability and temperature stability are other advantages. In addition, ferrites are more economically viable than most of other magnetic materials, and their magnetic and mechanical properties can be 'tailor made' as per the requirement of application.

Zinc ferrite $\left(\mathrm{ZnFe}_{2} \mathrm{O}_{4}\right)$ is a commercially important material and has been widely used as magnetic materials (Tanaka et al 1998; Sousa et al 2001; Grasset et al 2002; Kundu et al 2003a; Bid and Pradhan 2003; Ehrhardt et al 2003; Deng et al 2005), gas sensor (Niu et al 2004), catalysts (Toledo-Antonio et al 2002), photocatalysts (Qiu et al 2004), absorbent materials (Pineda et al 1997; Kobayashi et al 2002a, b; Tomás-Alonso and Palacios Latasa 2004; Ikenaga et al 2004) and so on. Zinc ferrite has been reported from high-temperature solid-state reaction, high-energy ball-milling (Bid and Pradhan 2003; Ehrhardt et al 2003; Shenoya et al 2004), sol-gel (Hamdeh et al 1997), hydrothermal technique (ToledoAntonio and Palacios Latasa 2002), coprecipitation (Kundu et al 2003b), rapid quenching (Tanaka et al 1998), liquid mix process (Kundu et al 2003a), thermal plasma (Mohair et al 2001), etc. However, some of these methods encountered problems such as the formation of 
undesirable phase, the requirement of complicated equipment or are time consuming due to multiple steps, etc. It is intriguing to develop an inexpensive, facile and fast method in the preparation of single phase zinc ferrite.

Self-propagating combustion synthesis is an exothermic redox reaction between metal nitrates (oxidizing agents) and an appropriate fuel (a reducing agent) and had been successfully used in synthesizing nanocrystalline metal oxides recently (Tao et al 1997). Compared with other methods, the combustion synthesis exhibits the following advantages: a good chemical homogeneity, simple equipment and preparation process, inexpensive raw materials, fast production rate, etc. In this paper, we report the synthesis of $\mathrm{ZnFe}_{2} \mathrm{O}_{4}$ nanoparticles through a self-propagation low-temperature combustion route employing metal oxalate precursors. In search of a suitable economic fuel, our use of polyethylene glycol (PEG) has given promising results in a single step. Some of the possible reasons for employing PEG in the present study are: PEG has a sharp melting point $\left(62^{\circ} \mathrm{C}\right)$ and decomposition temperature, and these are widely separated. The carboxylate precursors get well dispersed in the molten polyethylene glycol on its melting. PEG is known to be a good surfactant, and a dispersant and hence, ultrafine and nanomaterials are expected to be formed as the final products on combustion. PEG has alcoholic groups and it burns in air exothermically with precursors to form desired metal oxides/ferrites. In our earlier studies we have successfully used this fuel for the synthesis of $\gamma-\mathrm{Fe}_{2} \mathrm{O}_{3}$ and other metal oxides from different precursors (Venkataraman et al 2001; Lagashetty et al 2005; Basavaraja et al 2007). The structure and morphology of the as-prepared nanocrystalline $\mathrm{ZnFe}_{2} \mathrm{O}_{4}$ was characterized and its magnetic properties were studied. Our results show that self-propagating combustion is a fast and convenient method in the preparation of nanocrystalline $\mathrm{ZnFe}_{2} \mathrm{O}_{4}$.

\section{Experimental}

All the chemicals were of AR grade and were used as received. Double-distilled water was used for preparation of the required solutions.

\subsection{Preparation of metal oxalates}

An equimolar solution of ferrous ammonium sulphate heptahydrate/zinc nitrate and oxalic acid were dissolved in minimum quantity of water and was stirred well. The respective metal oxalate precipitate obtained was filtered through a sintered glass crucible and was washed with cold distilled water and alcohol until it was free from sulphate/nitrate ions and oxalic acid, and the filtrate was finally washed with dry acetone and then dried under vacuum.

\subsection{Preparation of $\mathrm{ZnFe}_{2} \mathrm{O}_{4}$}

The ferrous oxalate, zinc oxalate precursors were mixed with PEG in a weight ratio $1: 1: 5$, respectively, and ground well with a mortar and pestle. The resultant solid was placed in a silica crucible and heated in air. It was observed that initially PEG melted, then frothed and finally ignited to give $\mathrm{ZnFe}_{2} \mathrm{O}_{4}$ as a residue. On cooling to room temperature, no traces of carbon impurities were observed in the final residue of $\mathrm{ZnFe}_{2} \mathrm{O}_{4}$. As the reaction is fast, i.e. going to completion within $10 \mathrm{~min}$, and ignites auto-catalytically, the exact temperature of the reaction could not be measured. However, using a thermocouple the highest temperature of the reaction was found to be around $500^{\circ} \mathrm{C}$. The $\mathrm{ZnFe}_{2} \mathrm{O}_{4}$ was then characterized for its structure (employing XRD), morphology (employing SEM), particle size determination (employing TEM), etc.

\subsection{Characterization techniques}

Simultaneous thermogravimetric and differential scanning calorimetry (TG/DSC) traces were obtained from Mettlar Teledo Star instruments (Weinheim, Germany) under a dynamic flow of nitrogen at a flow rate of $100 \mathrm{ml} / \mathrm{min}$ and at a heating rate of $10^{\circ} \mathrm{C} / \mathrm{min}$. The XRD pattern was obtained employing a JEOL JDX-8p spectrometer using $\mathrm{CuK} \alpha$ radiation $(\lambda=1.54 \AA)$. The $\mathrm{X}$-ray generator was operated at $30 \mathrm{kV}$ and $20 \mathrm{~mA}$. The scanning range, $2 \theta$, was selected. The FT-IR spectrum of $\mathrm{ZnFe}_{2} \mathrm{O}_{4}$ nanoparticles was recorded on a Perkin-Elmer Spectrum One instrument in the range $4000-400 \mathrm{~cm}^{-1}$ at a resolution of $4 \mathrm{~cm}^{-1}$ by making $\mathrm{KBr}$ pellets. The scanning speed of $1 \% \mathrm{~min}$ and a chart speed of $20 \mathrm{~mm} / \mathrm{min}$ were used for the precise determination of the lattice parameters. The morphology of the nanoparticles was characterized using coupling JSM-840A scanning electron microscope. The electron microscope was operated at $20 \mathrm{kV}$. The sample was made conducting by the sputtering of gold using a Poloron DC 'sputtering unit' operated at $1.4 \mathrm{kV}$ and $18-20 \mathrm{~mA}$. Sample for TEM was prepared by placing a drop of the ethanol suspension of the nanoparticles on carbon-coated $\mathrm{Cu}$ TEM grids and subsequently drawing off excess solvent. The grids were examined using a Philips CM 200 transmission electron microscope operating at $200 \mathrm{kV}$. The preliminary magnetic measurements of the sample were recorded at room temperature on a Magneta, magnetic hysteresis loop tracer.

\section{Results and discussion}

Thermal analysis was performed to understand the combustion process used for the synthesis of the zinc ferrite sample. Simultaneous TG/DSC traces of the mixture of 


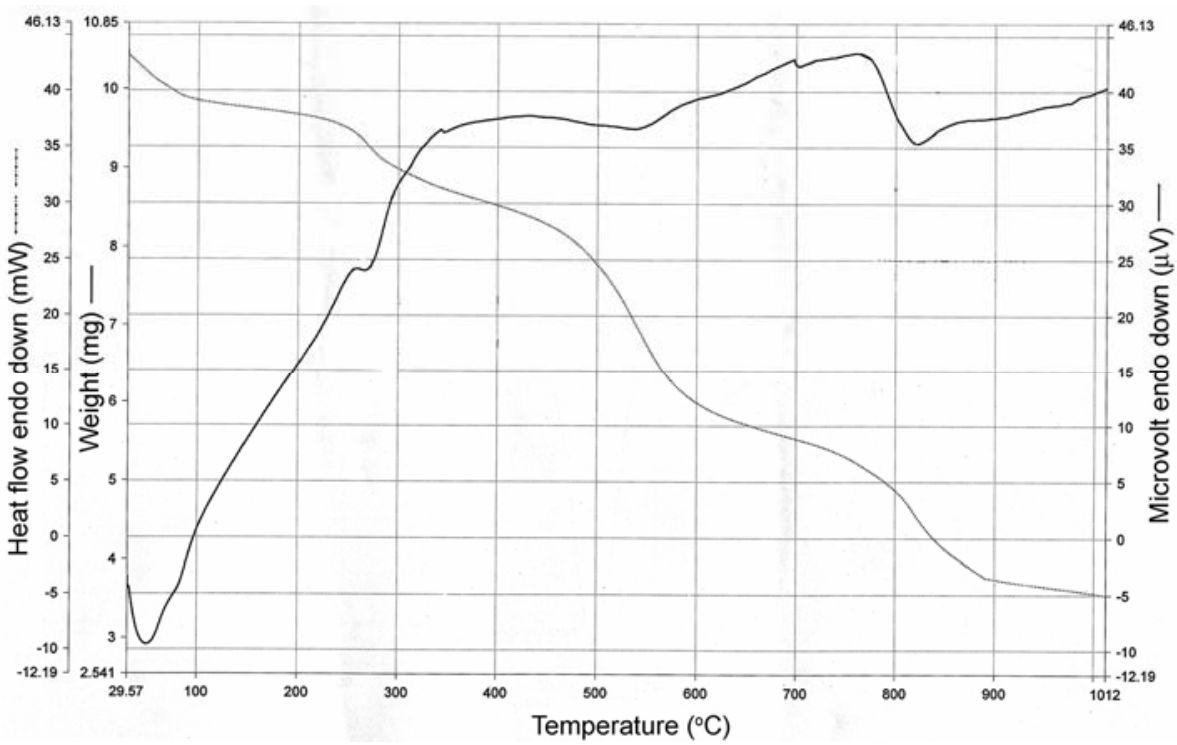

Figure 1. TG-DSC traces of precursors.

precursor (ferrous oxalate: zinc oxalate $1: 1$ ratio) is shown in figure 1 . The trace showed a multistep weight loss. The weight loss from room temperature to $100^{\circ} \mathrm{C}$ is attributed to desorption of water molecules present in the sample, which appear on the DSC curve as an endothermic peak at $60^{\circ} \mathrm{C}$. No considerable change in weight loss is observed from 100 to $240^{\circ} \mathrm{C}$. Thereafter, a continuous two step weight loss is noticed in the temperature ranging from 240 to $600^{\circ} \mathrm{C}$, showing a maximum weight loss, which also appeared as two broad endothermic peaks, at 273 and $540^{\circ} \mathrm{C}$ on the DSC trace. This weight loss is attributed to the decomposition of the oxalate precursor. The last broadened exothermic peak with small weight loss could be considered as a solid state reaction attributed to the gradual formation of zinc ferrite (Benito et al 2001). After $850^{\circ} \mathrm{C}$, no further distinguishable weight loss was detected, indicating that all organic constituents were eliminated. During the combustion, large amounts of gases such as $\mathrm{H}_{2} \mathrm{O}, \mathrm{CO}, \mathrm{CO}_{2}$ and $\mathrm{NO}$ are liberated (Mali and Ataie 2004).

The phase and purity of the as synthesized $\mathrm{ZnFe}_{2} \mathrm{O}_{4}$ was examined by X-ray diffraction (XRD) pattern and is shown in figure 2 . The intensity and ' $d$ ' values of the entire observed diffraction peaks perfectly match with the single-crystalline cubic spinel form of zinc ferrite (JCPDS Card No. 82-1049) having lattice constant $a=8.440 \AA$ with space group $\mathrm{Fd} 3 \mathrm{~m}$ (227). Absence of any peak from $\mathrm{ZnO}, \mathrm{Fe}_{2} \mathrm{O}_{3}$ or any other oxide phases confirmed the formation of phase pure zinc ferrite. According to the cubic crystal structure of spinel phase, the values of lattice parameter $a$ determined from the most intense (3 111$)$ reflection of XRD patterns using the formula $\left(1 / d_{h k l}^{2}=\left(h^{2}+k^{2}+l^{2}\right) / a^{2}, h k l=311\right)$ are $8.435 \AA$, respectively, well consistent with that of bulk $\mathrm{ZnFe}_{2} \mathrm{O}_{4}$. Elemental analysis by ICP-ES gives $\mathrm{Zn} / \mathrm{Fe}$ atomic ratio of
$0 \cdot 48-0 \cdot 51$ in as synthesized sample, indicating that this sample is indeed zinc ferrite with the chemical formula of $\mathrm{ZnFe}_{2} \mathrm{O}_{4}$.

The mean particle diameter was calculated from the

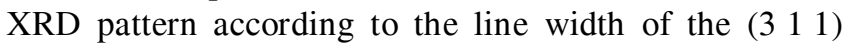
plane refraction peak using the following Debye-Scherrer equation (Cornell and Schwertmann 1996):

$$
D=\frac{K \lambda}{\beta_{1 / 2} \cos \theta} .
$$

The equation uses the reference peak width at angle $\theta$, where $\lambda$ is the X-ray wavelength ( $1.5418 \AA), \beta_{1 / 2}$ is the width of the XRD peak at half height and $K$ is the shape factor, about 0.9 for spherical shaped particles. The average crystallite size calculated from peak width is about $40 \mathrm{~nm}$, which is in accordance with the TEM results discussed later.

Fourier transform infrared (FT-IR) studies were performed to ascertain the metal-oxygen bonding. The FTIR spectrum of as synthesized $\mathrm{ZnFe}_{2} \mathrm{O}_{4}$ sample is shown in figure 3. The $\mathrm{ZnFe}_{2} \mathrm{O}_{4}$ shows the absorption in the region $3402,1620,630,555$ and $450 \mathrm{~cm}^{-1}$. The peaks at 630 and $555 \mathrm{~cm}^{-1}$ correspond to the metal-oxygen ( $\mathrm{Fe}-\mathrm{O}$ stretching vibrations) vibrational modes and corroborating the spinel structure characteristics of zinc ferrite (Rao et al 1970). This result is also in agreement with that of Waldron (Waldron 1955), assigned the high-frequency bands to the tetrahedral group (630 and $555 \mathrm{~cm}^{-1}$ region) and the low-frequency bands to octahedral group $\left(450 \mathrm{~cm}^{-1}\right)$. The peak at 3402 and $1620 \mathrm{~cm}^{-1}$ corresponds to water of hydration (Rao 1963). This was further supported by the weight loss measurements of thermogravimetric analysis. The peak at $1095 \mathrm{~cm}^{-1}$ is due to overtones. 


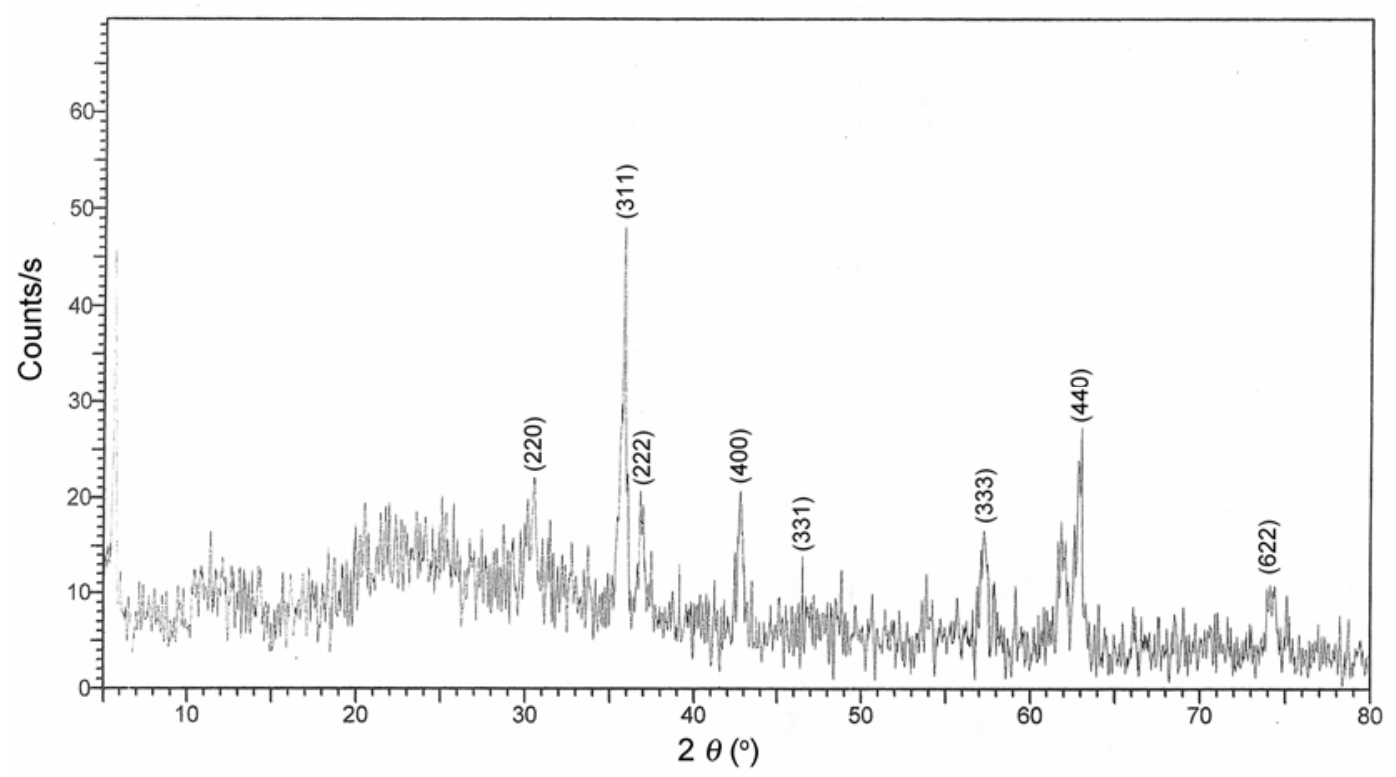

Figure 2. $\mathrm{XRD}$ pattern of $\mathrm{ZnFe}_{2} \mathrm{O}_{4}$.

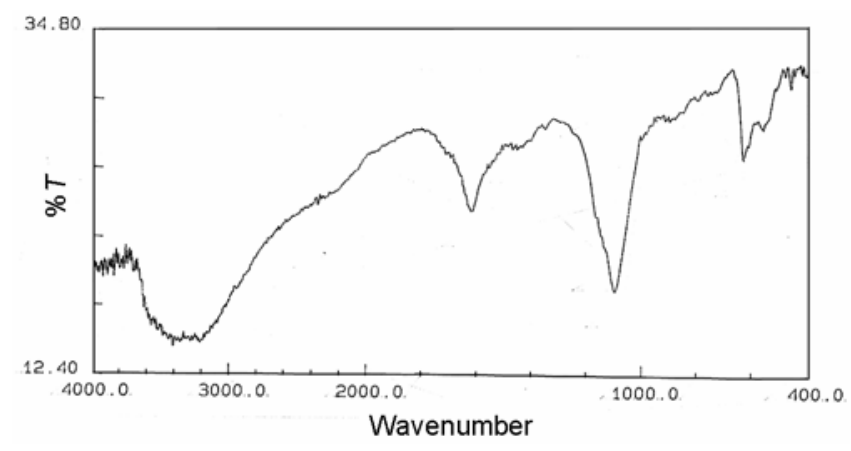

Figure 3. FT-IR spectrum of $\mathrm{ZnFe}_{2} \mathrm{O}_{4}$.

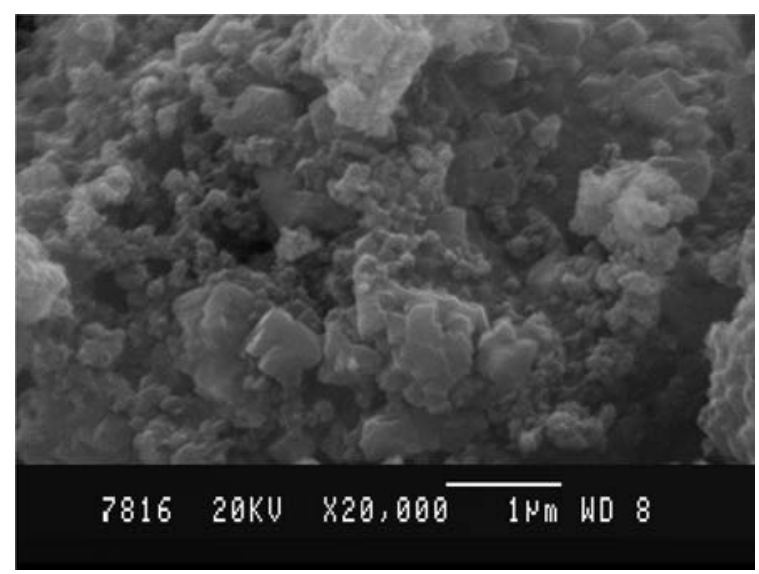

Figure 4. SEM image of $\mathrm{ZnFe}_{2} \mathrm{O}_{4}$.

The morphological characteristics of the obtained $\mathrm{ZnFe}_{2} \mathrm{O}_{4}$ were investigated by SEM and TEM analysis.
The SEM photograph of $\mathrm{ZnFe}_{2} \mathrm{O}_{4}$ sample (figure 4) reveals that sample exhibit a compact arrangement of homogeneous nanoparticles with roughly spherical shape. Most of the particles are aggregated, and so it is difficult to determine the exact size and shape of the particles. Hence, the exact size and shape of the $\mathrm{ZnFe}_{2} \mathrm{O}_{4}$ particles were examined by TEM images.

TEM was employed to visualize the size and shape and to confirm the nanocrystalline nature of the as synthesized zinc ferrite. Figure 5 shows the typical bright field TEM image of the as synthesized zinc ferrite nanoparticles. The TEM image clearly shows dense assembly of uniformly sized ferrite nanoparticles. The whole surface of the grid was covered with ferrute nanoparticles as shown in this image. It can be seen from the TEM image that the distribution of zinc ferrite nanoaprticles are polydispersed and are essentially spherical.

A particle size distribution histogram determined from TEM image is shown in figure 6. It can be seen that the mean particle size is ca. $45 \mathrm{~nm}$ with almost $93 \%$ of the particles are in the range $25-100 \mathrm{~nm}$, with a small percentage having diameters between 150 and $300 \mathrm{~nm}$. The particle sizes range from as low as $25 \mathrm{~nm}$ to as high as $300 \mathrm{~nm}$. The particle size distribution is broader and indicates the polycrystalline nature. The average particle size from the TEM and histogram is $45 \mathrm{~nm}$, which is also in accordance with the result calculated by the Scherrer formula.

The selected-area electron diffraction (SAED) pattern of the zinc ferrite is shown in figure 7. The ED pattern consists of concentric rings with spots over the rings. This feature indicates the samples are crystalline in nature (Wang et al 2001; Pawaskar et al 2002). 
Preliminary magnetic measurements were performed on the zinc ferrite nanoparticles with the use of magnetic hysteresis loop tracer. Figure 8 shows the hysteresis loop

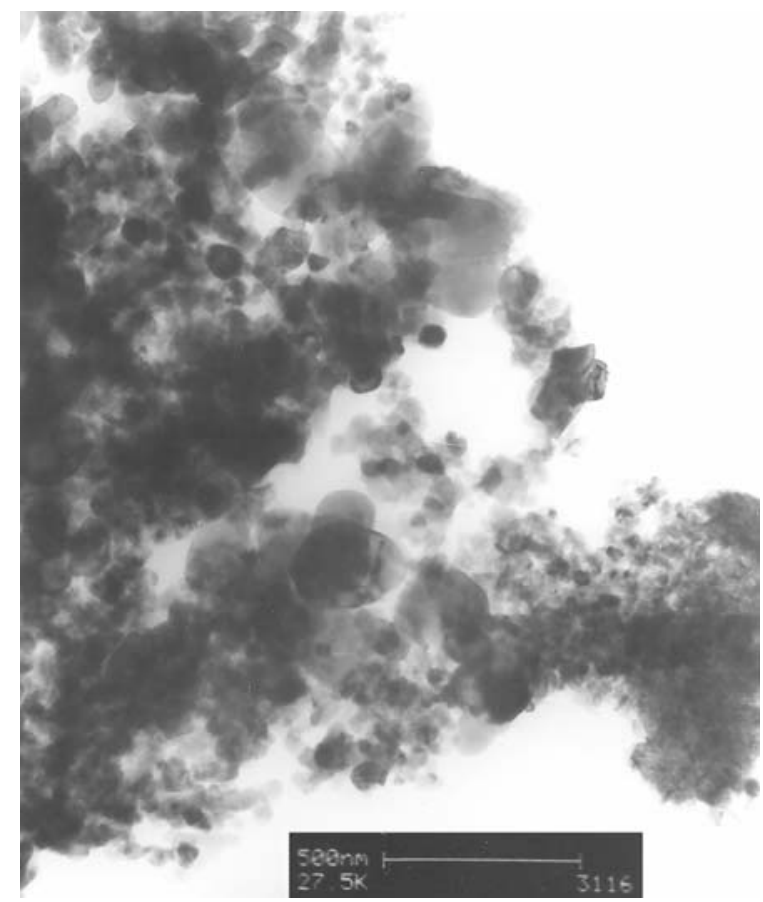

Figure 5. TEM image of $\mathrm{ZnFe}_{2} \mathrm{O}_{4}$.

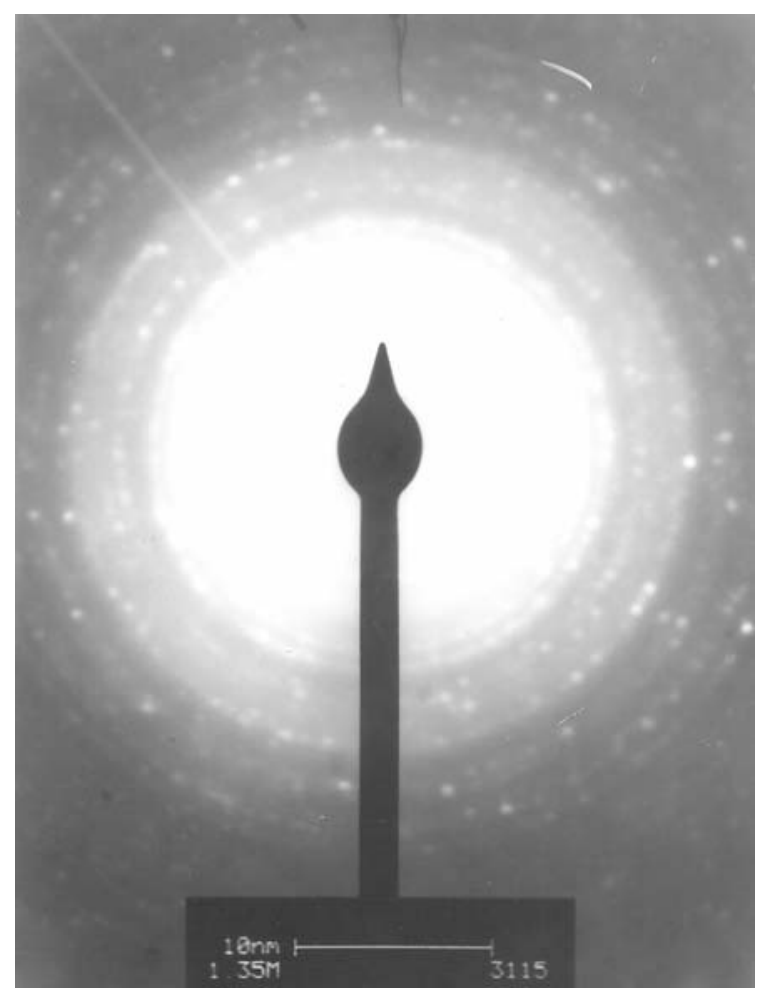

Figure 6. SAED pattern of $\mathrm{ZnFe}_{2} \mathrm{O}_{4}$. for the zinc ferrite nanoparticles measured at room temperature $(300 \mathrm{~K})$, in the range $\mathrm{H}= \pm 10 \mathrm{kOe}$. The saturation magnetization $\left(M_{\mathrm{s}}\right)$ obtained at room temperature was found to be $78.54 \mathrm{emu} / \mathrm{g}$, remanent magnetization $\left(M_{\mathrm{r}}\right)$ was $74.97 \mathrm{emu} / \mathrm{g}$ and coercivity $\left(H_{\mathrm{c}}\right)$ was $1297.52 \mathrm{Oe}$. The very large coercivity and low saturation magnetization at $300 \mathrm{~K}$ are consistent with a pronounced growth of magnetic anisotropy inhibiting the alignment of the moment in an applied field (Maaz et al 2007).

\section{Conclusions}

Self-propagating low-temperature synthesis offers a fast, relatively straightforward, single-step method for the synthesis of nanosized zinc ferrites. The characterization

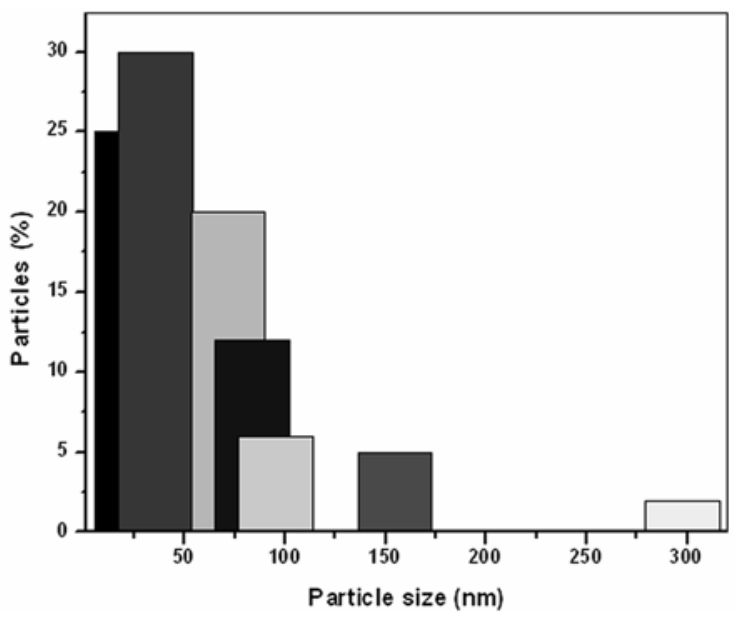

Figure 7. Particle size histogram of $\mathrm{ZnFe}_{2} \mathrm{O}_{4}$ derived from TEM image.

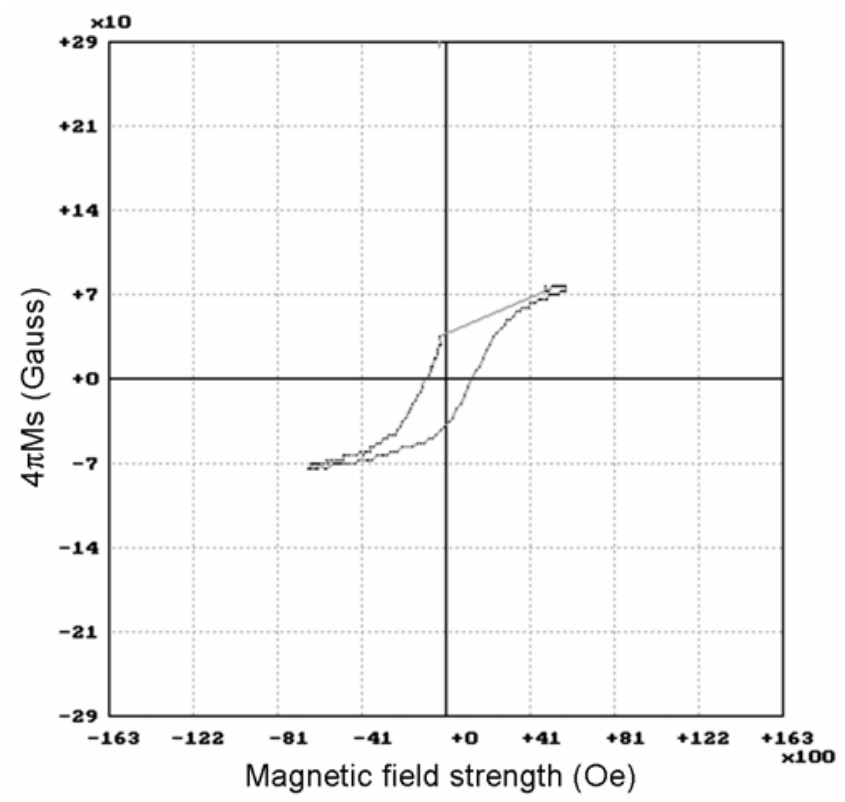

Figure 8. $\mathrm{MH}$ spectrum of $\mathrm{ZnFe}_{2} \mathrm{O}_{4}$. 
results show the formation of zinc ferrite with small nanoparticle sizes. The use of PEG as a fuel and as a controllable oxidant in the synthesis of zinc ferrite suggests that this combustion route is simple and can be applied for the synthesis of other ferrites and metal oxides. Because of the low cost of this process and the starting materials, this synthetic will be of interest to industries.

\section{Acknowledgements}

The authors wish to thank University Grants Commission (UGC), Innovative Programme, New Delhi, for financial assistance.

\section{References}

Basavaraja S, Vijayanand H, Venkataraman A, Deshpande U P and Shripathi T 2007 Synth. React. Inorg. Met-Org. NanoMetal Chem. 37409

Benito G, Morales M P, Requena J, Raposo V, Vazquez M and Moya J S 2001 J. Magn. Magn. Mater. 23465

Bhattacharyya A, Chakraborty P C, Mukherjee S, Mitra M K and Das G C 2001 Sci. Tech. Adv. Mater. 22449

Bid S and Pradhan S K 2003 Mater. Chem. Phys. 8227

Cornell R M and Schwertmann U 1996 The iron oxides: structure, properties, reactions, occurrence and uses ( $\mathrm{VCH}$ : Weinheim)

Deng H, Li X, Peng Q, Wang X, Chen J and Li Y 2005 Angew. Chem., Int. Ed. Engl. 442782

Ehrhardt H, Campbel S J and Hofmann M 2003 Scr. Mater. 48 1141

Grasset F, Labhsetwar N, Li D, Park D C, Saito N, Haneda H, Cador O, Roisnel T, Mornet S, Duguet E, Portier J and Etourneau J 2002 Langmuir 188209

Hamdeh H H, Ho J C, Oliver S A and Willey R J 1997 J. Appl. Phys. 811851

Ikenaga N, Ohgaito Y, Matsushima H and Suzuki T 2004 Fuel 83661

Jiles D C 1991 Introduction to magnetism and magnetic materials (London: Chapman and Hall) 2nd edn

Kobayashi M, Shirai H and Nunokawa M 2002a Energy Fuels 161378

Kobayashi M, Shirai H and Nunokawa M 2002b Ind. Eng. Chem. Res. 412903
Kondic L and Diez J A 2009 Phys. Rev. E79 026302

Kundu A, Anand S and Verma H C 2003a Powder Technol. 132 131

Kundu A, Upadhyay C and Verma H C 2003b Phys. Lett. A311 410

Lagashetty A, Havanoor V, Basavaraja S and Venkataraman A 2005 Bull. Mater. Sci. 28477

Maaz K, Mumtaz Arif, Hasanain S K and Ceylan Abdullah 2007 J. Magn. Magn. Mater. 308289

Mali A and Ataie A 2004 Ceram. Int. 301979

Martin C R 1994 Science 2661961

Mathew O S and Jiang R -S 2007 Chem. Eng. J. 12951

Mohair I, Szepvolgyi J, Bertoti I, Mohai M, Gubicaz J and Ungar T 2001 Solid State Ionics 141-142 163

Niu X, Du W and Du W 2004 Sens. Actuators B Chem. 99 405

Pawaskar N R, Sathaye S D, Bhadbhade M M and Patil K R 2002 Mater. Res. Bull. 371539

Pineda M, Palacios J M, Garcia E, Cilleruelo C and Lbarra J V 1997 Fuel 76567

Qiu J, Wang C and Gu M 2004 Mater. Sci. Eng., B, Solid-State Mater. Adv. Technol. 1121

Rao C N R 1963 Chemical applications of infrared spectroscopy (New York and London: Academic Press)

Rao G V S, Rao C N R and Ferraro J R 1970 Appl. Spectrosc. 24436

Shenoya S D, Joyb P A and Anantharaman M R 2004 J. Magn. Magn. Mater. 269217

Snelling E C 1989 Soft ferrites: properties and applications (London: Butterworth Publishing) 2nd edn

Sousa M H, Tourinho F A, Depeyrot J, Silva Joséda G, Cristina Maria and Lara F L 2001 J. Phys. Chem. B105 1168

Tanaka K, Makita M, Shimizugawa Y, Hirao K and Soga N 1998 J. Phys. Chem. Solids 591611

Tao Y, Zhao G, Zhang W and Xia S 1997 Mater. Res. Bull. 32 501

Toledo-Antonio J A, Nava N, Martínez M and Bokhimi X 2002 Appl. Catal., A Gen. 234137

Tomás-Alonso F and Palacios Latasa J M 2004 Fuel Process. Technol. 89191

Venkataraman A, Hiremath V A, Date S K and Kulkarni S D 2001 Bull. Mater. Sci. 24617

Waldron R D 1955 Phys. Rev. 991727

Wang Q, Yang H, Shi Jiunlin and Zou Guangtian 2001 Mater. Res. Bull. 36503

Willard M A, Kurihara L K, Carpenter E E, Calvin S and Harris V G 2004 Int. Mater. Rev. 49125 